\title{
Correction to: A 1-Year Prospective French Nationwide Study of Emergency Hospital Admissions in Children and Adults with Primary Immunodeficiency
}

\author{
Hélène Coignard-Biehler ${ }^{1,2,3} \cdot$ Nizar Mahlaoui ${ }^{2,3,4,5} \cdot$ Benoit Pilmis $^{1,2} \cdot$ Vincent Barlogis $^{6} \cdot$ Pauline Brosselin $^{2}$. \\ Nathalie De Vergnes ${ }^{2} \cdot$ Marianne Debré $^{4} \cdot$ Marion Malphettes $^{7} \cdot$ Pierre Frange $^{4,8,9} \cdot$ Emilie Catherinot $^{10}$. \\ Isabelle Pellier ${ }^{2,11}$ - Isabelle Durieu ${ }^{12}$. Antoinette Perlat ${ }^{13} \cdot$ Bruno Royer $^{14}$ - Alain Le Quellec ${ }^{15}$. Eric Jeziorski ${ }^{16}$. \\ Alain Fischer ${ }^{2,3,4,17}$. Olivier Lortholary ${ }^{1,2,3}$. for the CEREDIH French PID study group
}

Published online: 23 June 2020

(C) Springer Science+Business Media, LLC, part of Springer Nature 2020

Correction to: Journal of Clinical Immunology (2019) 39:702-712

https://doi.org/10.1007/s10875-019-00658-9

The original version of the article contained error regarding the presentation of the institutional authors in the PDF and $\mathrm{html}$ versions. Instead of having the corporate authorship under this umbrella: CEREDIH French PID study group, they all appeared in the author group section.

The correct version is displayed above.

Acknowledgments The European Society for Immunodeficiencies online database was used to collect the study data. The members of the CEREDIH French PID study group are (in alphabetical order) Laurent Aaron+, Daniel Adoue, Claire Aguilar, Nathalie Aladjidi, Alexandre Alcaïs, Zahir Amoura, Philippe Arlet, Corinne Armari-Alla, Brigitte Bader-Meunier, Sophie Bayart, Yves Bertrand, Boris Bienvenu, Stéphane Blanche, Damien Bodet, Bernard Bonnotte, Raphaël Borie, Patrick Boutard, Claire Briandet, Jean-Paul Brion, Jacques Brouard, Sarah Cohen-Beaussant, Laurence Costes, Louis-Jean Couderc, Pierre Cougoul, Virginie Courteille, Geneviève de Saint Basile, Catherine Devoldere, Anne Deville, Jean Donadieu, Eric Dore, Fabienne Dulieu, Christine Edan, Natacha Entz-Werle, Claire Fieschi, Amandine Forestier, Fanny Fouyssac, Vincent Gajdos, Lionel Galicier, Virginie Gandemer, Martine Gardembas, Catherine Gaud, Gaelle Guillerm, Eric Hachulla, Mohamed Hamidou, Olivier Hermine, Cyrille Hoarau, Sébastien
Humbert, Arnaud Jaccard, Serge Jacquot, Jean-Philippe Jais, Roland Jaussaud, Pierre-Yves Jeandel, Kamila Kebaili, Anne-Sophie Korganow, Olivier Lambotte, Fanny Lanternier, Claire Larroche, AnneSophie Lascaux, Emmanuelle Le Moigne, Vincent Le Moing, Yvon Lebranchu, Marc Lecuit, Guillaume Lefevre, Richard Lemal, Valérie Li-Thiao-Te, Aude Marie-Cardine, Nicolas Martin Silva, Agathe Masseau, Christian Massot, Françoise Mazingue, Etienne Merlin, Gérard Michel, Frédéric Millot, Béatrice Monlibert, Fabrice Monpoux, Despina Moshous, Luc Mouthon, Martine Munzer, Bénédicte Neven, Raphaëlle Nove-Josserand, Eric Oksenhendler, Marie OuachéeChardin, Caroline Oudot, Anne Pagnier, Jean-Louis Pasquali, Marlène Pasquet, Yves Perel, Capucine Picard, Christophe Piguet, Dominique Plantaz, Johan Provot, Pierre Quartier, Frédéric Rieux-Laucat, Pascal Roblot, Pierre-Marie Roger, Pierre-Simon Rohrlich, Hervé Rubie, Valéry Salle, Françoise Sarrot-Reynauld, Amélie Servettaz, Jean-Louis Stephan, Nicolas Schleinitz, Felipe Suarez, Laure Swiader, Sophie Taque, Caroline Thomas, Olivier Tournilhac, Caroline Thumerelle, François Tron, Jean-Pierre Vannier, Jean-François Viallard.

Publisher's Note Springer Nature remains neutral with regard to jurisdictional claims in published maps and institutional affiliations.
The online version of the original article can be found at https://doi.org/ 10.1007/s10875-019-00658-9

Olivier Lortholary

olivier.lortholary@aphp.fr

Extended author information available on the last page of the article 


\section{Affiliations}

Hélène Coignard-Biehler ${ }^{1,2,3} \cdot$ Nizar Mahlaoui $^{2,3,4,5} \cdot$ Benoit Pilmis $^{1,2} \cdot$ Vincent Barlogis $^{6} \cdot$ Pauline Brosselin $^{2}$. Nathalie De Vergnes ${ }^{2} \cdot$ Marianne Debré $^{4} \cdot$ Marion Malphettes $^{7} \cdot$ Pierre Frange $^{4,8,9} \cdot$ Emilie Catherinot $^{10}$. Isabelle Pellier 2,11 . Isabelle Durieu ${ }^{12}$. Antoinette Perlat ${ }^{13} \cdot$ Bruno Royer $^{14}$ - Alain Le Quellec ${ }^{15}$. Eric Jeziorski ${ }^{16}$. Alain Fischer ${ }^{2,3,4,17}$. Olivier Lortholary ${ }^{1,2,3} \cdot$ for the CEREDIH French PID study group

1 Service de Maladies Infectieuses et Tropicales, Centre d'Infectiologie Necker Pasteur, Hôpital Universitaire NeckerEnfantsMalades, Assistance Publique-Hôpitaux de Paris, Université de Paris, Paris, France

2 Centre de Référence Déficits Immunitaires Héréditaires (CEREDIH), Hôpital Universitaire Necker-Enfants Malades, Assistance Publique-Hôpitaux de Paris, Paris, France

3 Institut Imagine, Université de Paris, 149 Rue de Sèvres, 75015 Paris, France

4 Unité d'Immuno-Hématologie et Rhumatologie Pédiatrique, Hôpital Universitaire Necker-Enfants Malades, Assistance PubliqueHôpitaux de Paris, Paris, France

5 Laboratoire de Biostatistiques, Hôpital Universitaire Necker-Enfants Malades, Assistance Publique-Hôpitaux de Paris, Paris, France

6 Assistance Publique-Hôpitaux de Marseille, Service de Pédiatrie et Hématologie Pédiatrique, CHU de Marseille, Hôpital de la Timone, Marseille, France

7 Assistance Publique-Hôpitaux de Paris, Immunologie-Hématologie, Hôpital Saint Louis, Paris, France
8 Laboratoire de Microbiologie Clinique, Hôpital Universitaire Necker-Enfants Malades, Assistance Publique-Hôpitaux de Paris, Paris, France

9 Unité de Recherche EA 7327, Université de Paris, Paris, France

10 Hôpital Foch, Service de Pneumologie, Suresnes, France

11 Immunologie-Hématologie-Oncologie Pédiatrique, Centre Hospitalo-Universitaire Angers, Angers, France

12 Centre Hospitalo-Universitaire Lyon, Service de Médecine Interne et Pathologie Vasculaire, Centre Hospitalier Lyon Sud, PierreBénite, France

13 Service de Médecine Interne, Centre Hospitalo-Universitaire Pontchaillou, Rennes, France

14 Service d'Hématologie Clinique et de Thérapie Cellulaire, Centre Hospitalo-Universitaire, Amiens, France

15 Service de Médecine Interne, Centre Hospitalo-Universitaire, Montpellier, France

16 Service de Pédiatrie Générale, Infectiologie et Immunologie Clinique, Centre Hospitalo-Universitaire, Montpellier, France

17 Collège de France, Paris, France 